\title{
The Trial-Ready Cohort for Preclinical/Prodromal Alzheimer's Disease (TRC-PAD) - A Fundamental Ally in AD Prevention Research
}

\author{
A.P. Porsteinsson, E.D. Clark \\ Department of Psychiatry, University of Rochester School of Medicine and Dentistry, Rochester, NY, USA

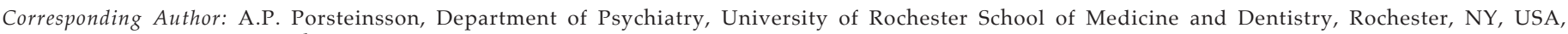 \\ Anton_Porsteinsson@URMC.Rochester.edu

A lzheimer's disease (AD) remains one of our greatest unmet medical needs, without any approved disease-modifying therapies. The emotional and financial burden of AD is enormous and predicted to grow exponentially with increasing median population age, posing a major public health problem. The potential to prevent or improve cognitive decline due to $\mathrm{AD}$ has important implications. There are medications currently approved for symptomatic treatment of AD, but they have limited clinical benefits and do not change the ultimate trajectory of the disease. The need to find effective treatments for $\mathrm{AD}$ that can prevent, slow, arrest, or even reverse the disease is ever more urgent and interventions that delay the symptomatic onset of $\mathrm{AD}$ would have a major public health impact (1).

The best opportunity to modify the course of $\mathrm{AD}$ is prior to the onset of widespread neuronal damage and emergence of clinical symptomatology. The concept of preclinical and prodromal Alzheimer's Disease has rapidly evolved with recent advances in biomarker and imaging techniques that can detect evidence of $\mathrm{AD}$ pathology in the brains of healthy individuals without evidence of significant cognitive or functional impairment (2). Abnormalities in amyloid production, accumulation, or toxic sequelae of amyloid-beta $(A \beta)$ species drives disease onset but the extent of amyloid in the brain does not correlate with disease progression. Conversely, there is compelling evidence that tau burden tracks better with clinical emergence of $\mathrm{AD}(3)$.

Initiating therapies in the preclinical/prodromal stages of $\mathrm{AD}$ may offer greater efficacy in preventing or slowing the disease course as this allows participants to be treated prior to the plateau of fibrillary $A \beta$, extensive appearance of tau (neurofibrillary) pathology, neuroinflammation, and irreversible synaptic and neuronal loss. With this changing perspective, a new era of AD "secondary prevention" trials has emerged, aimed at preventing the progression of clinical symptoms in individuals in whom the pathophysiological process of AD has already begun. Regrettably, the majority of $\mathrm{AD}$ focused studies are delayed because there are too few participants (4). $\mathrm{AD}$ prevention trials require an expansive screening and enrollment funnel and improving recruitment methods is a critical priority for the AD field $(5,6)$. The timeframe, complexity, and expense of the recruitment process and site activation for these secondary prevention trials are extremely challenging, and site start-up and trial enrollment represent a significant bottleneck for drug development in AD. It is likely that future early-stage prevention trials will move even earlier in the process of amyloid accumulation and have to utilize remote electronic contact and assessments in order to screen large number of individuals and follow participants over long periods of time, requiring an overhaul the current clinical trial recruitment and assessment process. In the USA and internationally, several AD-focused participant recruitment registries exist but the field is still gathering data on best practices to optimize the registries aiming to recruit, engage, and retain greater numbers of, and more diverse qualified study participants (5).

In this issue of the Journal, we see five articles $(7,8,9,10,11)$ that describe the rationale, design, execution, limitations of, and key lessons learned from the TrialReady Cohort for Preclinical/Prodromal Alzheimer's Disease (TRC-PAD) Project. TRC-PAD is an ambitious program, addressing wide-ranging issues in participant recruitment and site management. The rationale for the project's design is to develop and utilize the APT Webstudy and the TRC-PAD cohort to accelerate AD prevention trial enrollment as current methods of recruitment for prevention studies are slow and have high screen fail rates $(\sim 80 \%)$ adding years to study duration. TRC-PAD builds on existing efforts in informatics, web-based consent, innovative cognitive assessments, biostatistics, and multiple existing registry programs. The feeder registries (Brain Health Registry, Alzheimer's Prevention Registry, Alzheimer's Association TrialMatch, University of California, Irvine (UCI) Consent-to-Contact Registry, and Cleveland Clinic Healthy Brains Registry) filter participants based on age and cognitive status and invite selected individuals not meeting criteria for dementia to enroll in the APT Webstudy, which in turn conducts unsupervised, web-based capture of demographic, medical, lifestyle and genetic factors, as well as longitudinal web-based cognitive testing and symptom questionnaires, to assess 
participants' risk of elevated brain amyloid. The initial risk algorithm is derived from analysis of existing study data and then iteratively updated through an appropriate regression-based statistical approach to biomarker testing results.

The individuals at highest risk within the APT Webstudy pool of asymptomatic (preclinical) and very mildly symptomatic (prodromal) individuals are referred for in-person clinical and biomarker assessments at select AD clinical trial sites. Participants identified with biomarker profiles indicative of AD pathology, including asymptomatic individuals with minimally elevated or "intermediate" levels of amyloid, are eligible for the in-clinic trial-ready cohort (TRC-PAD), where they are followed at semi-annual in-person visits until they are ready for enrollment in preclinical/prodromal therapeutic trials, while continuing quarterly, remote, web-based cognitive assessments in the APT Webstudy. Biomarker-negative individuals continue remote webbased follow-up in the APT Webstudy. If longitudinal trajectories and changes to other risk factors indicate increasing amyloid risk, those participants are eligible for repeat in-person clinical and biomarker assessment. Participants with known biomarker status can be directly referred to the in-clinic TRC cohort and are encouraged to participate in the APT Webstudy.

Taken together, the overarching goal of this project is to accelerate current and future secondary prevention trial enrollment through an innovative, highly efficient approach to identify, evaluate, and enroll appropriate preclinical and prodromal trial candidates, supported by a new site network with enhanced capacities for more efficient and effective conduct of AD clinical trials to help the field accelerate the evaluation of investigational prevention therapies. The identification of participants with biomarker profiles indicative of $\mathrm{AD}$ pathology serves as a prognostic enrichment strategy to select individuals with a greater likelihood of having substantial worsening in cognition, within a reasonable timeframe, that can be practically assessed within the setting of a clinical trial. Future challenges will be to enhance APT webstudy recruitment strategies and tactics, facilitate enrollment of underrepresented racial and ethnic groups, as well as engaging and maintaining current participants.

Conflict of interests: Dr. Porsteinsson reports personal fees from Acadia Pharmaceuticals, Avanir, Cadent Therapeutics, Eisai, Functional Neuromodulation, and Tetra Discovery Partners; grants to his institution from Avanir, Biogen, Biohaven, Eisai, Eli Lilly, Genentech/Roche, Novartis, outside of the submitted work. Dr. Clark has nothing to disclose.

\section{References}

1. Rafii MS, Aisen PS. Alzheimer's Disease Clinical Trials: Moving Toward Successful Prevention. CNS Drugs. 2019;33(2):99-106.

2. Jack CR, Jr., Albert MS, Knopman DS, et al. Introduction to the recommendations from the National Institute on Aging-Alzheimer's Association workgroups on diagnostic guidelines for Alzheimer's disease. Alzheimers Dement. 2011;7(3):257-262.

3. Cummings J, Blennow K, Johnson K, et al. Anti-Tau Trials for Alzheimer's Disease: A Report from the EU/US/CTAD Task Force. Journal of the Prevention of Alzheimer's Disease. J Prev Alzheimers Dis. 2019;6(3):157-163. doi: 10.14283/jpad.2019.14.

4. Fargo KN, Carrillo MC, Weiner MW, Potter WZ, Khachaturian Z. The crisis in recruitment for clinical trials in Alzheimers and dementia: An action plan for solutions. Alzheimers Dement 2016;12(11):1113-1115.

5. Langbaum JB, High N, Nichols J, Kettenhoven C, Reiman EM, Tariot PN. The Alzheimer's Prevention Registry: a large internet-based participant recruitment registry to accelerate referrals to Alzheimer's-focused studies. J Prev Alz Dis 2020;4(7):242-250

6. Together we make the difference: National strategy for recruitment and participation in Alzheimer's and related dementias clinical research National Institutes of Health 2018 Oct. Retrieved from https://www.nia.nih.gov/ sites / default / files / 2018-10/alzheimers-disease-recruitment-strategy-final.pdf

7. Aisen PS, Sperling RA, Cummings J, et al. The Trial-Ready Cohort for Preclinical/prodromal Alzheimer's Disease (TRC-PAD) Project: An Overview. J Prev Alz Dis 2020;4(7):208-212

8. Walter S, Langford O, Clanton T, et al. The Trial-Ready Cohort for Preclinical/ Prodromal Alzheimer's disease (TRC-PAD): Experience from the first 3 years. J Prev Alz Dis 2020;4(7):234-241

9. Walter S, Clanton T, Langford O, et al. Recruitment into the Alzheimer Prevention Trials (APT) Webstudy for a Trial-Ready Cohort for Preclinical and Prodromal Alzheimer's Disease (TRC-PAD). J Prev Alz Dis 2020;4(7):219-225

10. Jimenez-Maggiora GA, Bruschi S, Langford O, et al. TRC-PAD: Accelerating Recruitment of AD Clinical Trials through Innovative Information Technology. J Prev Alz Dis 2020;4(7):226-233

11. Langford O, Raman R, Sperling RA, et al. Predicting amyloid burden to accelerate recruitment of secondary prevention clinical trials. J Prev Alz Dis 2020;4(7):213-218 